\title{
Quantum nonlocality in the excitation energy transfer in the Fenna-Matthews-Olson complex
}

\author{
Charlotta Bengtson, Michael Stenrup ${ }^{\dagger \ddagger}$ Erik Sjöqvist ${ }^{\S}$
}

July 31, 2018

\begin{abstract}
The Fenna-Matthews-Olson (FMO) complex - a pigment protein complex involved in photosynthesis in green sulfur bacteria - is remarkably efficient in transferring excitation energy from light harvesting antenna molecules to a reaction center. Recent experimental and theoretical studies suggest that quantum coherence and entanglement may play a role in this excitation energy transfer (EET). We examine whether bipartite quantum nonlocality, a property that expresses a stronger-than-entanglement form of correlation, exists between different pairs of chromophores in the FMO complex when modeling the EET by the hierarchically coupled equations of motion method. We compare the results for nonlocality with the amount of bipartite entanglement in the system. In particular, we analyze in what way these correlation properties are affected by different initial conditions. It is found that bipartite nonlocality only exists when the initial conditions are chosen in an unphysiological manner and probably is absent when considering the EET in the FMO complex in its natural habitat. It is also seen that nonlocality and entanglement behave quite differently in this system. In particular, for localized initial states, nonlocality only exists on a very short time scale and then drops to zero in an abrupt manner. As already known from previous studies, quantum entanglement between chromophore pairs on the other hand is oscillating and exponentially decaying and follow thereby a pattern more similar to the chromophore population dynamics. The abrupt disappearance of nonlocality in the presence of nonvanishing entanglement is a phenomenon we call nonlocality sudden death; a striking manifestation of the difference between these two types of correlations in quantum systems.
\end{abstract}

*Department of Chemistry - Ångström Laboratory, Theoretical Chemistry, Uppsala University, Box 538, SE-751 21 Uppsala, Sweden

${ }^{\dagger}$ Department of Chemistry - Angström Laboratory, Theoretical Chemistry, Uppsala University, Box 538, SE-751 21 Uppsala, Sweden

${ }_{\ddagger}^{\ddagger}$ Uppsala Center for Computational Chemistry - $\mathrm{UC}_{3}$, Uppsala University, Box 538, SE-751 21 Uppsala, Sweden

${ }^{\S}$ Department of Physics and Astronomy, Uppsala University, Box 516, SE-751 20 Uppsala, Sweden 


\section{INTRODUCTION}

In recent years, there has been an increasing interest in studying biological systems in terms of the existence of nontrivial quantum effects ${ }^{1 / 8}$. Especially the excitation energy transfer (EET) between chromophores in photosynthetic complexes has been heavily studied ever since quantum coherence between excited states in the Fenna-Matthews-Olson (FMO) complex was experimentally verified by two dimensional electronic spectroscopy ${ }^{2}$. Not only was the existence of coherent pathways in the FMO complex revealed, quantum coherence was also shown to last much longer than expected for such large and noisy systems, and a hypothesis is that this may play a role in the known very efficient EET in photosynthetic

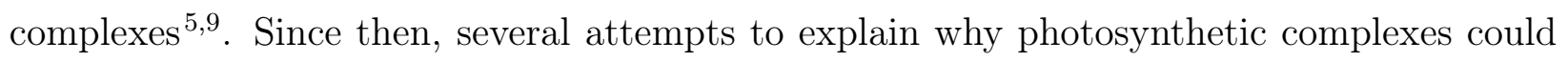
benefit from coherent EET have been made ${ }^{78}$. One such is that the quantum coherence could help to direct the energy flow towards the reaction center in a unidirectional manner ${ }^{318}$.

The experimental evidence for coherent EET in the FMO complex motivates the development of new methods to model the excited state dynamics of chromophores in a surrounding protein scaffold. It has become clear that Markovian models cannot fully capture the behaviour of the system-environment interactions in these systems and hence non-Markovian models have to be considered. Based on the hierarchial expansion technique proposed in Refs. $\frac{10111}{1}$, Ishizaki and Fleming refined the theoretical framework and developed a tool for investigating the excited state dynamics in a photosynthetic complex ${ }^{3 \mid 12}$. This form of hierarchially coupled equations of motions (HEOM) has served as the benchmarking method for these systems since it is able to describe quantum coherent motion and incoherent electron hopping in a unified manner ${ }^{12}$. Using this method, Sarovar et al. ${ }^{[5}$ examined quantum entanglement in the FMO complex and found that bipartite entanglement of chromophores exists on a time scale relevant for the mechanism of EET. Since entanglement can be used as a resource in quantum information processing tasks, it was speculated if the findings could play a role in explaining the near unity efficiency for these complexes to convert solar light into chemical energy.

Quantum nonlocality, as introduced by Bell ${ }^{13}$, is another correlation property of composite quantum systems. While quantum entanglement, already mentioned by Schrödinger 
as the key property that distinguishes quantum mechanics from the classical world 14 , is a meaningful property only for quantum systems, nonlocality is given by a criterion that is formed without specifying whether the systems are quantum mechanical or not. In the quantum-mechanical context, although entanglement and nonlocality are the same for pure states, this is not in general true for mixed states, i.e., they are inequivalent properties for

composite quantum systems $\frac{15 / 16}{}$. In particular, nonlocality is a stronger correlation property than entanglement in the sense that nonlocality implies entanglement but not vice versa ${ }^{17}$.

The aim of this study is to examine whether bipartite nonlocality can exist during EET in the FMO complex, which would imply a striking nonclassical feature of this process. By studying the existence of quantum nonlocality in such a complex, it is possible to add insights into if and how quantum effects play a role in photosynthesis; insights that could be of interest for artificial photosynthesis and solar cells as well as for quantum computation. In recent studies $\frac{1819}{}$, a considerable enhancement in the efficiency due to quantum coherence was found in model systems of photosynthesis mimicking solar cells. If such discoveries also can be connected to the existence of entanglement and nonlocality, it would be desirable to explore the underlying physics of such phenomena further.

\section{MODELING THE EET IN THE FMO COMPLEX}

EET in the FMO complex has been modeled by employing the HEOM method ${ }^{3[12}$. A description of the FMO complex as well as the quantities and conditions used to model EET, is given followed by a brief review of the HEOM based model of the FMO complex, modified by including an explicit mechanism of the excitation energy trapping at the reaction center.

\section{The FMO complex}

The FMO complex is a photosynthetic complex found in green sulfur bacteria, Chlorobaculum tepidum. It consists of three identical monomers, each containing seven chromophores embedded in a protein scaffold, as can be seen in Fig. 1. The structure of this complex as 


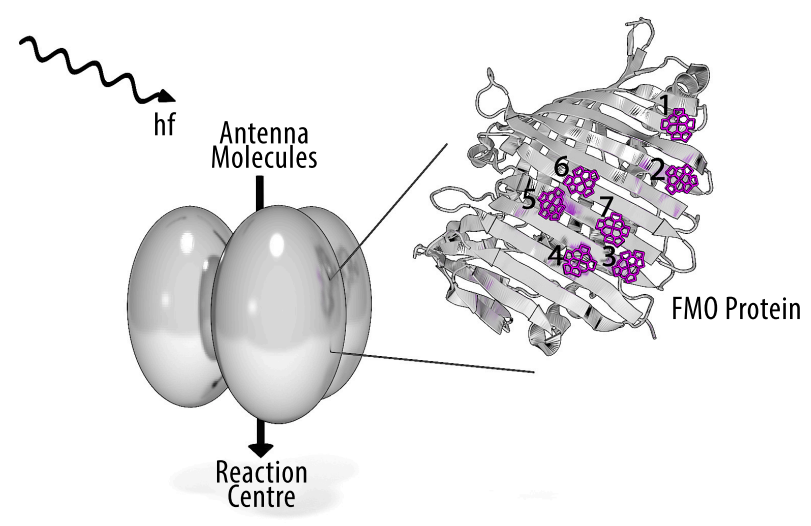

Figure 1: The FMO complex trimer and its position between the antenna molecules and the reaction center to the left, and the chromophores in their protein scaffold for one monomer to the right. The chromophores are chlorophylls in this particular system.

well as its site excitation energies and inter-site couplings have been experimentally investigated by different spectroscopic methods 220121 and the system has been used as a model for larger light harvesting complexes for more than 35 years $^{22}$.

The FMO complex does not include any antenna molecules. In other words, it is not responsible for capturing the light energy, but only for transferring it to the reaction center. The antenna molecules are located in such a way that they can transfer excitation energy to the three monomer units. We restrict the system studied to one monomer; a reasonably simplifying assumption in the description of correlations accompanying EET in this system.

The individual chromophores in the FMO complex can be modeled as two level systems (TLSs) by taking into account only the $S_{0} \rightarrow S_{1}$ transition, with $S_{0}$ and $S_{1}$ being the singlet ground state and singlet first excited state, respectively. Furthermore, since Green sulfur bacteria recieve very little sunlight in their natural habitat ${ }^{5}$, it is reasonable to assume that the FMO complex only contains one such excitation at a time. This reduces the electronic Hamiltonian of the FMO complex (one monomer) to have the form

$$
\hat{H}_{e}=\sum_{k=1}^{7} E_{k}|k\rangle\left\langle k\left|+\sum_{k \neq l}^{7} J_{k l}\right| k\right\rangle\langle l|,
$$

where $E_{k}$ is the electronic excitation energy of chromophore $k$ when being in its excited state, while the other chromophores remain in their ground state. This corresponds to the 
localized state

$$
|k\rangle=\left|S_{0}^{1} \ldots S_{1}^{k} \ldots S_{0}^{7}\right\rangle
$$

where the superscript denotes the chromophore site. Furthermore, $J_{k l}$ is the dipole coupling describing the electrostatic interaction between the charge distribution of chromophores $k$ and $l$. It depends strongly on the dipole moment orientations and relative positions of the chromophores in the protein complex structure ${ }^{1}$.

Calculations suggest that the most favourable structure is that chromophores 3 and 4 are linked directly to the reaction center ${ }^{1}$. This result has been confirmed experimentally when examining the FMO complex with masspectrometry 21 . Since the structure of the FMO is known ${ }^{1}$, this implies that chromophores 1 and 6 connect to the base plate (that is the part of the antenna molecule complex that connects it to the FMO). The simplest way to model EET in the FMO complex would hence be to assume a localized excitation on either chromophore 1 or 6 as the initial conditions for EET. This can be written as

$$
\hat{\varrho}_{\text {localized }}^{x}=|x\rangle\langle x|, \quad x=1,6 .
$$

However, since the distance between the antenna molecules and the base plate as well as the distance between the base plate and the FMO are so large $\left(\sim 2 \mathrm{~nm}^{23} \sqrt{25}\right.$ and $\sim 1.5 \mathrm{~nm}^{23}$, respectively) in comparison to the intracomplex distances, a model that better captures the condition for initial excitation of the FMO complex in its natural habitat is to assume that the excitation is transferred from the base plate to the FMO by Förster resonance energy transfer $(\mathrm{FRET})^{26}$. This would populate the FMO exciton states $\left|e_{r}\right\rangle$, being the eigenstates of the Hamiltonian in Eq. (1), in proportion to their occurrence at chromophore 1 or 6. These FRET initial states can hence be written as

$$
\hat{\varrho}_{\mathrm{FRET}}^{x}=\sum_{r=1}^{7}\left|\left\langle x \mid e_{r}\right\rangle\right|^{2}\left|e_{r}\right\rangle\left\langle e_{r}\right|, \quad x=1,6 .
$$

In this work, we shall examine correlations between chromophore pairs in EET arising from the initial states given by Eqs. (3) and (4). 


\section{The HEOM method for the FMO complex}

$\mathrm{HEOM}^{\frac{3 \pi 12}{12}}$ is a numerically exact method in which the environmental influence on a quantum system is treated in a statistical manner. For the FMO complex, the system of interest is the seven chromophores in one of the monomers with the Hamiltonian given in Eq. (1). The protein scaffold is modeled as a set of harmonic oscillator modes, i.e., a phonon bath, which represents nuclear motion, both intramolecular and those of the environment. The transfer of excitation energy from one chromophore to another occurs via nonequilibrium nuclear configuration (phonon states) in accordance with a vertical Franck-Condon transition. The phonons, locally and linearly coupled to each chromophore $k$, relax to their equilibrium states under energy loss. This so-called reorganization energy, denoted $\lambda_{k}$, can be measured via the Stokes shift $\underline{3227}$.

The memory of the local environment of chromophore $k$ is characterized by a relaxation function $\Gamma_{k}(t)$, modeled as an overdamped Brownian oscillator, which takes the form

$$
\Gamma_{k}(t)=2 \lambda_{k} e^{-\gamma_{k} t}
$$

The parameter $\gamma_{k}$ represents the time scale of the fluctuations and energy dissipation for the $k$ th chromophore and is related to the non-Markovian behaviour of the dynamics. The relaxation function, and hence $\gamma_{k}$, can be investigated by two dimensional electronic spectroscopy $3 \sqrt[327]{ }$.

The time dependent density operator $\hat{\varrho}(t)$ describing EET in the FMO complex is obtained by solving a set of hierchically coupled equations of motion,

$$
\frac{\partial}{\partial t} \hat{\zeta}(\mathbf{n}, t)=-\left(i \mathcal{L}_{e}+\sum_{k=1}^{7} n_{k} \gamma_{k}\right) \hat{\zeta}(\mathbf{n}, t)+\sum_{k=1}^{7}\left[\Phi_{k} \hat{\zeta}\left(\mathbf{n}_{k+}, t\right)+n_{k} \Theta_{k} \hat{\zeta}\left(\mathbf{n}_{k-}, t\right)\right]
$$

where the operator $\hat{\zeta}(\mathbf{0}, t)$ is identical to $\hat{\varrho}(t)$ while the higher order operators $\hat{\zeta}(\mathbf{n} \neq \mathbf{0}, t)$ are auxiliary operators. Here, $\mathbf{n}$ is a set of nonnegative integers, $\mathbf{n}=\left(n_{1}, n_{2}, . ., n_{7}\right)$. The notation $\mathbf{n}_{k+}\left(\mathbf{n}_{k-}\right)$ stands for adding (substracting) 1 to the corresponding $n_{k}$ in $\mathbf{n}$. The Liouvillian superoperator $\mathcal{L}_{e}$ is composed of the electronic Hamiltonian of the FMO complex and the site reorganization energies, and takes the form

$$
\mathcal{L}_{e} \hat{g}=\left[\hat{H}_{e}+\sum_{k=1}^{7} \lambda_{k}|k\rangle\langle k|, \hat{g}\right]
$$


with $[\cdot, \cdot]$ the commutator and $\hat{g}$ any linear operator acting nontrivially on the single excitation subspace of the full Hilbert space of the FMO complex. The superoperators $\Phi_{k}$ and $\Theta_{k}$ are so called phonon-induced relaxation operators and correspond to the influence of the environmental fluctuations. They have the form

$$
\Phi_{k} \hat{g}=i\left[\hat{V}_{k}, \hat{g}\right]
$$

and

$$
\Theta_{k} \hat{g}=i \frac{2 \lambda_{k}}{\beta \hbar^{2}}\left[\hat{V}_{k}, \hat{g}\right]+\frac{\lambda_{k}}{\hbar} \gamma_{k}\left\{\hat{V}_{k}, \hat{g}\right\}
$$

with $\hat{V}_{k}=|k\rangle\langle k|$ and $\{\cdot, \cdot\}$ the anti-commutator. Here, $\beta=\left(k_{B} T\right)^{-1}$ with $k_{B}$ being the Boltzmann constant and $T$ the temperature of the phonon bath.

The HEOM can be truncated by setting

$$
\frac{\partial}{\partial t} \hat{\zeta}(\mathbf{n}, t)=-i \mathcal{L}_{e} \hat{\zeta}(\mathbf{n}, t)
$$

for all $\mathbf{n}$ satisfying $n_{1}+n_{2}+\ldots+n_{7}=N, N$ being the truncation level. This condition will terminate the generation of auxiliary operators.

Following Ref. 28 , a Liouvillian that models the trapping of excitation of chromophores 3 and 4 is added to the right-hand side of Eq. (6). It has the form

$$
\mathcal{L}_{\text {trap }} \hat{\zeta}(\mathbf{n}, t)=-r_{\text {trap }}\{|3\rangle\langle 3|, \hat{\zeta}(\mathbf{n}, t)\}-r_{\text {trap }}\{|4\rangle\langle 4|, \hat{\zeta}(\mathbf{n}, t)\}
$$

where $r_{\text {trap }}$ is the trapping rate, assumed to be the same for chromophores 3 and 4 . When comparing to Eq. (6), it can be seen that adding this Liouvillian makes the population of chromophore 3 and 4 decay faster than for the other chromophores.

The density operator describing EET in the FMO complex can be written as

$$
\hat{\varrho}(t)=\sum_{k=1}^{7} \varrho_{k k}(t)|k\rangle\langle k|+\sum_{k=1}^{7} \sum_{l>k}^{7}\left(\varrho_{k l}(t)|k\rangle\left\langle l\left|+\varrho_{k l}^{*}(t)\right| l\right\rangle\langle k|\right),
$$

where $|k\rangle$ are the site basis states defined in Eq. (2). Here, $\varrho_{k k}$ is the population of an excitation at chrompohore $k$ and $\varrho_{k l}$ describes the coherence between chromophores $k$ and $l$. Note that $\hat{\varrho}$ can be viewed as a $7 \times 7$ dimensional Hermitian matrix due to the restriction to one coherent excitation at each instant of time. 


\section{MEASURE OF BIPARTITE QUANTUM CORRELATIONS}

Quantum nonlocality is a property of composite quantum systems whose subsystems show correlations that are too strong to be explained by a local realistic theory 29 , i.e., a theory where physical variables (such as, e.g., positions and momenta of particles) are assumed to have well-defined local values prior to measurement. The existence of quantum nonlocality was discovered when Bell derived ${ }^{13}$ an upper bound (Bell's inequality) for correlations between two systems to be local, and then showed that the correlations within certain composite quantum systems may exceed this limit.

Since Bell's original formulation of his inequality, there have been other Bell-like inequalities. One such that is suitable for investigating bipartite nonlocality for TLSs like the chromophores in the FMO complex is the Clauser-Horne-Shimony-Holt (CHSH) inequality 30. It states that any set of variables $a, a^{\prime}, b$, and $b^{\prime}$ that can take values \pm 1 must satisfy

$$
\left|\langle a b\rangle+\left\langle a b^{\prime}\right\rangle+\left\langle a^{\prime} b\right\rangle-\left\langle a^{\prime} b^{\prime}\right\rangle\right| \leq 2
$$

if a local realistic theory applies to the pairs $a, a^{\prime}$ and $b, b^{\prime}$ at distant locations. Here, $\langle a b\rangle$ denotes the average of the product of the outcomes $a$ and $b$.

To test the validity of the CHSH inequality for measurements on two distant TLSs, each characterized by the Pauli operators $\hat{\boldsymbol{\sigma}}=\left(\hat{\sigma}_{1}, \hat{\sigma}_{2}, \hat{\sigma}_{3}\right)$, described as a composite quantum system with density operator $\hat{\rho}$, the operator

$$
\begin{aligned}
\hat{\mathfrak{B}}_{\mathrm{CHSH}}= & (\mathbf{a} \cdot \hat{\boldsymbol{\sigma}}) \otimes(\mathbf{b} \cdot \hat{\boldsymbol{\sigma}})+(\mathbf{a} \cdot \hat{\boldsymbol{\sigma}}) \otimes\left(\mathbf{b}^{\prime} \cdot \hat{\boldsymbol{\sigma}}\right) \\
& +\left(\mathbf{a}^{\prime} \cdot \hat{\boldsymbol{\sigma}}\right) \otimes(\mathbf{b} \cdot \hat{\boldsymbol{\sigma}})-\left(\mathbf{a}^{\prime} \cdot \hat{\boldsymbol{\sigma}}\right) \otimes\left(\mathbf{b}^{\prime} \cdot \hat{\boldsymbol{\sigma}}\right)
\end{aligned}
$$

with $\mathbf{a}, \mathbf{a}^{\prime}, \mathbf{b}$, and $\mathbf{b}^{\prime}$ unit vectors in $R^{3}$, can be used, as the measurements of $(\mathbf{a} \cdot \hat{\boldsymbol{\sigma}}),\left(\mathbf{a}^{\prime} \cdot \hat{\boldsymbol{\sigma}}\right),(\mathbf{b}$. $\hat{\boldsymbol{\sigma}})$, and $\left(\mathbf{b}^{\prime} \cdot \hat{\boldsymbol{\sigma}}\right)$ on the respective TLSs have outcomes \pm 1 . Thus, by comparing with Eq. (13), one may conclude that the correlation in $\hat{\rho}$ is nonlocal, i.e., cannot be accounted for by any local realistic theory, if there exists $\mathbf{a}, \mathbf{a}^{\prime}, \mathbf{b}$, and $\mathbf{b}^{\prime}$ such that $\left|\left\langle\hat{\mathfrak{B}}_{\mathrm{CHSH}}\right\rangle\right|=\left|\operatorname{Tr}\left(\hat{\rho} \hat{\mathfrak{B}}_{\mathrm{CHSH}}\right)\right|>2$.

A necessary and sufficient condition for the correlation between any two TLSs to be

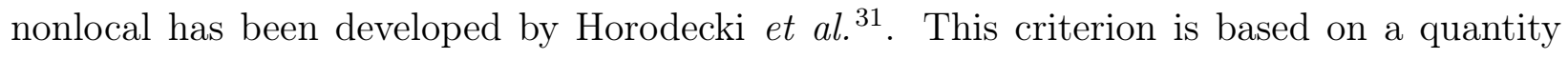
$M(\hat{\rho})$ that maximizes the expectation value of the Bell operator in Eq. (14) such that

$$
\max _{\mathbf{a}, \mathbf{a}^{\prime}, \mathbf{b}, \mathbf{b}^{\prime}}\left|\left\langle\hat{\mathfrak{B}}_{\mathrm{CHSH}}\right\rangle\right|=2 \sqrt{M(\hat{\rho})} .
$$


$M(\hat{\rho})$ is found as

$$
M(\hat{\rho})=\mu_{p}+\mu_{q}
$$

where $\mu_{p}$ and $\mu_{q}$ are the two greatest eigenvalues of $|T(\hat{\rho})|^{2}, T(\hat{\rho})$ being the correlation matrix

$$
T(\hat{\rho})=\left(\begin{array}{lll}
t_{11} & t_{12} & t_{13} \\
t_{21} & t_{22} & t_{23} \\
t_{31} & t_{32} & t_{33}
\end{array}\right)
$$

with matrix elements $t_{\alpha \beta}=\operatorname{Tr}\left(\hat{\rho} \hat{\sigma}_{\alpha} \otimes \hat{\sigma}_{\beta}\right)$ for all combinations of Pauli operators $\hat{\sigma}_{\alpha}$. The correlation is nonlocal whenever $1<M(\hat{\rho}) \leq 2$, where the maximum value is given by the Cirel'son bound ${ }^{\sqrt[32]{2}}\left|\left\langle\hat{\mathfrak{B}}_{\mathrm{CHSH}}\right\rangle\right| \leq 2 \sqrt{2}$. This motivates that the quantity ${ }^{15}$

$$
B(\hat{\rho})=\sqrt{\max \{(M(\hat{\rho})-1), 0\}}
$$

can be used as a measure of the amount of nonlocality. This Bell-CHSH measure is directly comparable with concurrence, defined as 33

$$
C(\hat{\rho})=\max \left\{\lambda_{1}-\lambda_{2}-\lambda_{3}-\lambda_{4}, 0\right\}
$$

where $\lambda_{1}, \ldots \lambda_{4}$ are the decreasingly ordered eigenvalues of the positive operator

$$
\hat{R}=\sqrt{\sqrt{\hat{\rho}} \sigma_{2} \otimes \sigma_{2} \hat{\rho}^{*} \sigma_{2} \otimes \sigma_{2} \sqrt{\hat{\rho}}}
$$

with complex conjugation taken in the computational product basis. Concurrence uniquely determines the entanglement of formation $\frac{34}{34}$ of two TLSs; as such, $C(\hat{\rho})$ is an entanglement measure in its own right. It has been used to analyze the amount of bipartite entanglement in the FMO complex by Sarovar et al. $\stackrel{5}{5}$.

The time evolution of the composite system of chromophores $n$ and $m$ in the FMO complex can be found by calculating the reduced density operator from the full density operator $\hat{\varrho}$ given in Eq. (12). This is done by tracing $\hat{\varrho}$ over the other five chromophore degrees of freedom. The resulting reduced density operator takes the form

$$
\begin{aligned}
\hat{\rho}_{m n}=\operatorname{Tr}_{r \neq n, m} \hat{\varrho}= & \sum_{k, l, p, q=0,1} \rho_{k l ; p q}^{m n}\left|S_{k}^{m} S_{l}^{n}\right\rangle\left\langle S_{p}^{m} S_{q}^{n}\right| \\
= & {\left[\operatorname{Tr}(\hat{\varrho})-\left(\varrho_{m m}+\varrho_{n n}\right)\right]\left|S_{0}^{m} S_{0}^{n}\right\rangle\left\langle S_{0}^{m} S_{0}^{n}\right| } \\
& +\varrho_{m m}\left|S_{0}^{m} S_{1}^{n}\right\rangle\left\langle S_{0}^{m} S_{1}^{n}\left|+\varrho_{m n}\right| S_{0}^{m} S_{1}^{n}\right\rangle\left\langle S_{1}^{m} S_{0}^{n}\right| \\
& +\varrho_{m n}^{*}\left|S_{1}^{m} S_{0}^{n}\right\rangle\left\langle S_{0}^{m} S_{1}^{n}\left|+\varrho_{n n}\right| S_{1}^{m} S_{0}^{n}\right\rangle\left\langle S_{1}^{m} S_{0}^{n}\right|,
\end{aligned}
$$


where we have taken into account that HEOM and excitation trapping are not trace preserving, i.e., that $\operatorname{Tr}(\hat{\varrho}) \leq 1$. Note that $\hat{\rho}_{m n}$ can be viewed as a $4 \times 4$ Hermitian matrix. One finds

$$
\mu_{1}=\mu_{2}=4\left|\varrho_{m n}\right|^{2}, \quad \mu_{3}=\left[\operatorname{Tr}(\varrho)-2\left(\varrho_{m m}+\varrho_{n n}\right)\right]^{2},
$$

which implies

$$
B\left(\hat{\rho}_{m n}\right)=\sqrt{\max \left\{\left(\max \left\{8\left|\varrho_{m n}\right|^{2}, 4\left|\varrho_{m n}\right|^{2}+\left[\operatorname{Tr}(\hat{\varrho})-2\left(\varrho_{m m}+\varrho_{n n}\right)\right]^{2}\right\}-1\right), 0\right\}} .
$$

Similarly, concurrence for this class of states takes the form

$$
C\left(\hat{\rho}_{m n}\right)=2\left|\varrho_{m n}\right|
$$

which coincides with the square root of $\mu_{1}$.

Recent attempts to quantify the amount of coherence in quantum states ${ }^{35136}$ have lead to different types of coherence measures. One of these is the $l_{1}$ norm measure of coherence ${ }^{36}$, defined as

$$
\mathcal{C}_{l_{1}}(\hat{\rho})=\sum_{k \neq l}\left|\rho_{k l}\right|
$$

i.e., the sum of the absolute value of the off-diagonal elements of the density operator. For $\hat{\rho}_{m n}$ this reduces to

$$
\mathcal{C}_{l_{1}}\left(\hat{\rho}_{m n}\right)=2\left|\varrho_{m n}\right|
$$

which implies that concurrence and the $l_{1}$ norm measure of coherence are in fact identical quantities for all chromophore pairs. Thus, a nonzero concurrence can equally well be interpreted as an expression for having a nonzero coherence, rather than being a sign of correlation. This is a consequence of the restriction to the single excitation subspace of the Hamiltonian in Eq. (1). Thus, the restriction itself implies the existence of entanglement as a consequence of the nonvanishing coherence in the system.

Nonlocality, on the other hand, is essentially different from coherence as it also depends on the populations $\varrho_{m m}$ and $\varrho_{n n}$ of the chromophores via the nondegenerate eigenvalue $\mu_{3}$ of $|T(\hat{\rho})|^{2}$. This makes the Bell-CHSH measure a proper quantifier of correlation between chromophore pairs. 
We may ask under what circumstances a chromophore pair exhibiting nonvanishing coherence is nonlocally correlated. As follows from the explicit expressions in Eq. (23), the nonlocality condition relies on the relation between the populations $\varrho_{m m}$ and $\varrho_{n n}$ of the pair and the coherence $2\left|\varrho_{m n}\right|$. Since the latter is bounded by the former as $\frac{37}{37}$

$$
\left|\varrho_{m n}\right| \leq \sqrt{\varrho_{m m} \varrho_{n n}}
$$

which follows from positivity of the reduced density matrix, we see that $\mu_{1}$ and $\mu_{2}$ become very small unless the system is considerably localized to the $(m, n)$ pair, in case of which $\mu_{3}$ also becomes large. Alternatively, $\mu_{3}$ can be close to the critical value 1 sufficient for nonlocal correlations if $\varrho_{m m}$ and $\varrho_{n n}$ are both very small (in the order of a few \%, say) and $\operatorname{Tr}(\hat{\varrho}) \lesssim 1$; however, this cannot give rise to any nonlocality. To see this, we first note that

$$
\begin{aligned}
M\left(\rho_{m n}\right) & =\mu_{1}+\mu_{3} \leq 4 \varrho_{m m} \varrho_{n n}+\left(\operatorname{Tr}(\varrho)-2 \varrho_{m m}-2 \varrho_{n n}\right)^{2} \\
& =\operatorname{Tr}(\hat{\varrho})\left(\operatorname{Tr}(\varrho)-4 \varrho_{m m}-4 \varrho_{n n}\right)+4 \varrho_{m m}^{2}+4 \varrho_{n n}^{2}+12 \varrho_{m m} \varrho_{n n} \\
& \lesssim 1-4\left(\varrho_{m m}+\varrho_{n n}-\varrho_{m m}^{2}-\varrho_{n n}^{2}-3 \varrho_{m m} \varrho_{n n}\right)
\end{aligned}
$$

by combining Eqs. (22), (27), and $\operatorname{Tr}(\hat{\varrho}) \lesssim 1$. It is straightforward to see that $\varrho_{m m}+\varrho_{n n}-$ $\varrho_{m m}^{2}-\varrho_{n n}^{2}-3 \varrho_{m m} \varrho_{n n} \geq 0$ for small $\varrho_{m m}$ and $\varrho_{n n}$, which implies that

$$
M\left(\rho_{m n}\right) \leq 1
$$

excluding the possibility of nonlocal correlations in this case. We conclude that only chromphore pairs for which the population is large can be nonlocally correlated.

\section{NUMERICAL DETAILS}

The parameters of our numerical model are chosen in accordance with previous work on EET in the FMO complex. As the electronic FMO Hamiltonian for Chlorobaculum tepidum 
in the chromophore site basis, we use ${ }^{1}$

$$
H_{e}=\left(\begin{array}{ccccccc}
200 & -87.7 & 5.5 & -5.9 & 6.7 & -13.7 & -9.9 \\
-87.7 & 320 & 30.8 & 8.2 & 0.7 & 11.8 & 4.3 \\
5.5 & 30.8 & 0 & -53.5 & -2.2 & -9.6 & 6.0 \\
-5.9 & 8.2 & -53.5 & 110 & -70.7 & -17.0 & -63.3 \\
6.7 & 0.7 & -2.2 & -70.7 & 270 & 81.1 & -1.3 \\
-13.7 & 11.8 & -9.6 & -17.0 & 81.1 & 420 & 39.7 \\
-9.9 & 4.3 & 6.0 & -63.3 & -1.3 & 39.7 & 230
\end{array}\right) \text {, }
$$

where all numbers are in units of $\mathrm{cm}^{-1}$ with a total offset of $12210 \mathrm{~cm}^{-1}$. The reorganization energy $\lambda_{k}$ and the relaxation time-scale $\gamma_{k}$ are assumed to have the same values, $35 \mathrm{~cm}^{-1}$ and $50 \mathrm{fs}^{-1}\left[\sqrt[38]{ }\right.$, respectively, for all seven chromophores ${ }^{3}$. The time scale $r_{\text {trap }}^{-1}$ for the trapping by the reaction center is set to $1 \mathrm{ps}^{\left[\frac{1139}{139}\right.}$ and the bath temperature to $300 \mathrm{~K}$ (same as in Ref. ${ }^{[3}$ ).
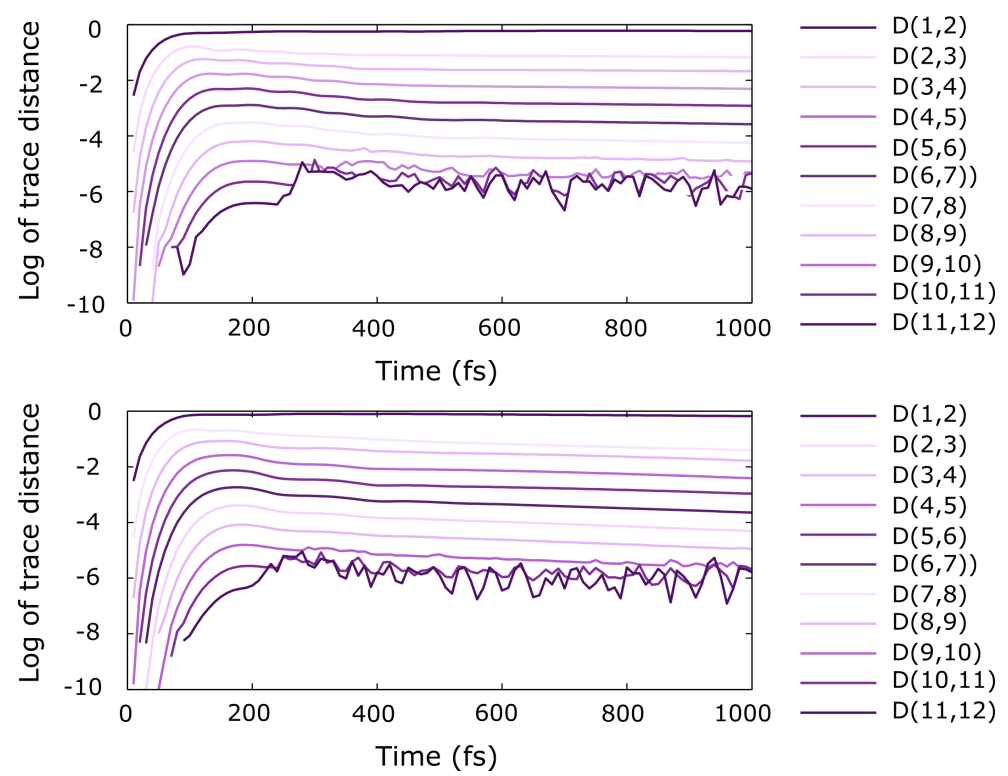

Figure 2: Convergence of the density operator with respect to the truncation level of the HEOM. The change in the density operator when going from level $N$ to level $N+1$ is characterized by the logarithm of the corresponding trace distance $\mathrm{D}(N, N+1) \equiv D\left(\hat{\varrho}_{N}, \hat{\varrho}_{N+1}\right)=$ $\frac{1}{2} \operatorname{Tr}\left|\hat{\varrho}_{N}-\hat{\varrho}_{N+1}\right|$. The initial excitation is either on chromophore 1 (upper panel) or chromophore 6 (lower panel). Notice how at the higher truncation levels $(N \gtrsim 10)$ the trace distance is comparable to the numerical noise. 
The HEOM given by Eq. (6) is numerically integrated in the range 0 to 1000 fs using the Runge-Kutta-Dormand-Prince method 40 . To measure the convergence of the HEOM solution, we use the trace distance ${ }^{41}$

$$
D\left(\hat{\varrho}_{N}, \hat{\varrho}_{N+1}\right)=\frac{1}{2} \operatorname{Tr}\left|\hat{\varrho}_{N}-\hat{\varrho}_{N+1}\right|
$$

for density operators $\hat{\varrho}_{N}$ and $\hat{\varrho}_{N+1}$ at truncation level $N$ and $N+1$, respectively. In our simulations in the next section, we use a truncation level $N=12$, which implies an accuracy in the order of $10^{-5}$, as can be seen from Fig. 2 .

\section{RESULTS AND DISCUSSION}

Our simulations show that no nonlocality is found when the initial states are given by Eq. (4), corresponding to FRET from the antenna molecules to the FMO, although entanglement still exists. In particular, a considerable amount of quantum entanglement is found for chromophore 1 and 2 when the exciton states are projected on chromophore 1 as well as for chromophore 5 and 6 when the exciton states are projected on chromophore 6 . These results can be seen in Figs. 3 and 4 . On the other hand, bipartite quantum nonlocality exists when the localized initial conditions according to Eq. (3) are used. For these initial conditions, nonlocality is found for two chromophore pairs; chromophore 1 and 2 when chromophore 1 is initially excited, and chromophore 5 and 6 when chromophore 6 is initially excited. These results are presented in Figs. 5 and 6 together with the time dependence of the bipartite entanglement for the same pairs of chromophores.

To get futher insights into these findings we analyze in more detail the structure of the evolution arising from the two types of initial conditions. Let us start with the FRET case, where the initial states are given by Eq. (44). By writing the exciton states $\left|e_{r}\right\rangle=\sum_{k=1}^{7} c_{r k}|k\rangle$, we find

$$
\hat{\varrho}_{\mathrm{FRET}}^{x}=\sum_{r=1}^{7} c_{r x}^{2}\left|e_{r}\right\rangle\left\langle e_{r}\right|
$$

where we have used that all $c_{r k}$ are real-valued and we have ordered $\left|e_{r}\right\rangle$ with increasing energy. The reduced density matrix for the $(m, n)$ pair is characterized by the site probablities 


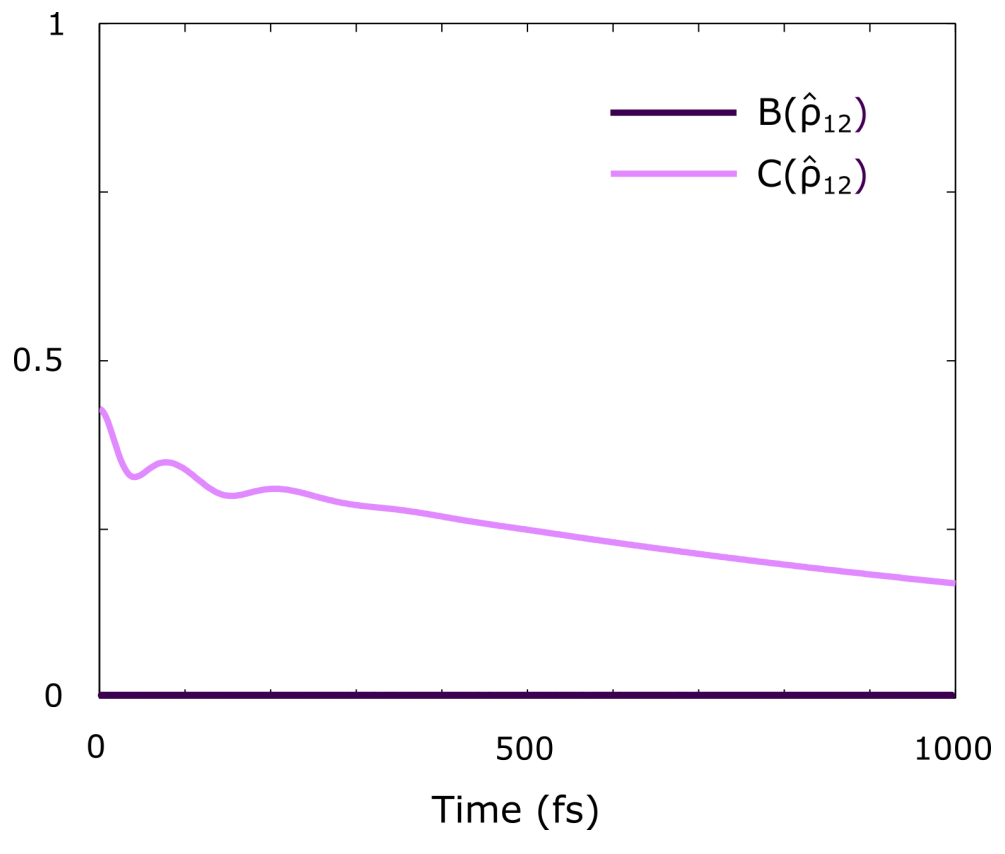

Figure 3: Nonlocality $B\left(\hat{\rho}_{12}\right)$ and entanglement $C\left(\hat{\rho}_{12}\right)$ for the chromophore pair 1 and 2 as a function of time for FRET initial state when exciton states projected on chromophore 1 is used as initial condition. Note that the nonlocality $B\left(\hat{\rho}_{12}\right)$ vanishes at all times.

$\varrho_{m m}, \varrho_{n n}$, and the off-diagonal term $\varrho_{m n}$ given by

$$
\begin{aligned}
\varrho_{m m} & =\sum_{r=1}^{7} c_{r x}^{2} c_{r m}^{2}, \\
\varrho_{n n} & =\sum_{r=1}^{7} c_{r x}^{2} c_{r n}^{2}, \\
\varrho_{m n} & =\sum_{r=1}^{7} c_{r x}^{2} c_{r m} c_{r n} .
\end{aligned}
$$

We focus on the $x=1$ case. As demonstrated above, only chromphore pairs for which the population is large can exhibit nonlocal correlations; thus, we only need to consider the dominant terms in the density operator of the FMO complex. By inspection of the explicit eigenvectors, we find that $\hat{\varrho}_{\mathrm{FRET}}^{x=1}$ is dominated by the pure state components $\left|e_{3}\right\rangle$ and $\left|e_{6}\right\rangle$. In fact, $c_{31}^{2} \approx 0.769$ and $c_{61}^{2} \approx 0.208$; thus, these two exciton states populate almost $98 \%$ of this initial state and we can safely ignore all the other exciton states. We further find that both $\left|e_{3}\right\rangle$ and $\left|e_{6}\right\rangle$ are to a large extent localized to the first two chromophores, as can be 


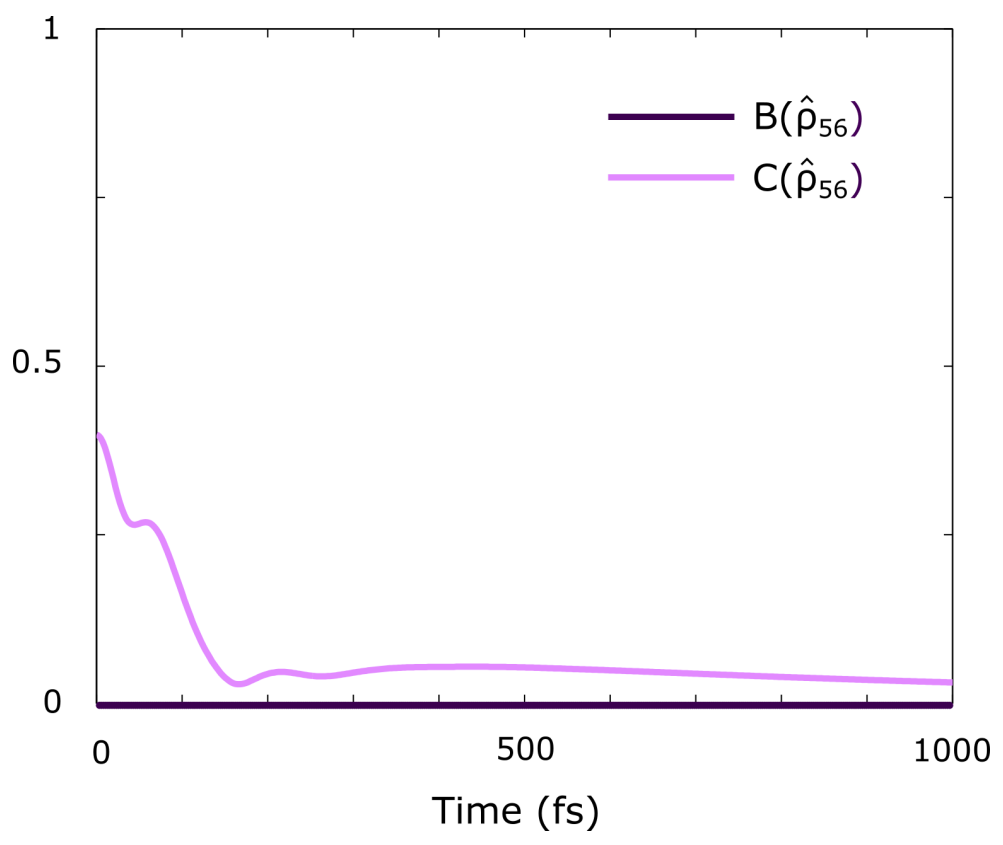

Figure 4: Nonlocality $B\left(\hat{\rho}_{56}\right)$ and entanglement $C\left(\hat{\rho}_{56}\right)$ for the chromophore pair 5 and 6 as a function of time for FRET initial state when exciton states projected on chromophore 6 is used as initial condition. Note that the nonlocality $B\left(\hat{\rho}_{12}\right)$ vanishes at all times.

seen from the expansion coefficients

$$
\begin{aligned}
& c_{31}=0.877, \quad c_{32}=0.440, \\
& c_{61}=-0.456, \quad c_{62}=0.871,
\end{aligned}
$$

obtained by numerical diagonalization of the electronic Hamiltonian $H_{e}$. Thus, correlation is essentially concentrated to the first two chromophores, and can be expressed in terms of the matrix elements

$$
\begin{aligned}
\varrho_{12} & \approx c_{31}^{3} c_{32}+c_{61}^{3} c_{62} \\
& \equiv c_{31}^{2}\left|c_{31} c_{32}\right|-c_{61}^{2}\left|c_{61} c_{62}\right|=0.214 \\
\varrho_{11} & \approx c_{31}^{4}+c_{61}^{4}=0.635 \\
\varrho_{22} & \approx c_{31}^{2} c_{32}^{2}+c_{61}^{2} c_{62}^{2}=0.307 .
\end{aligned}
$$




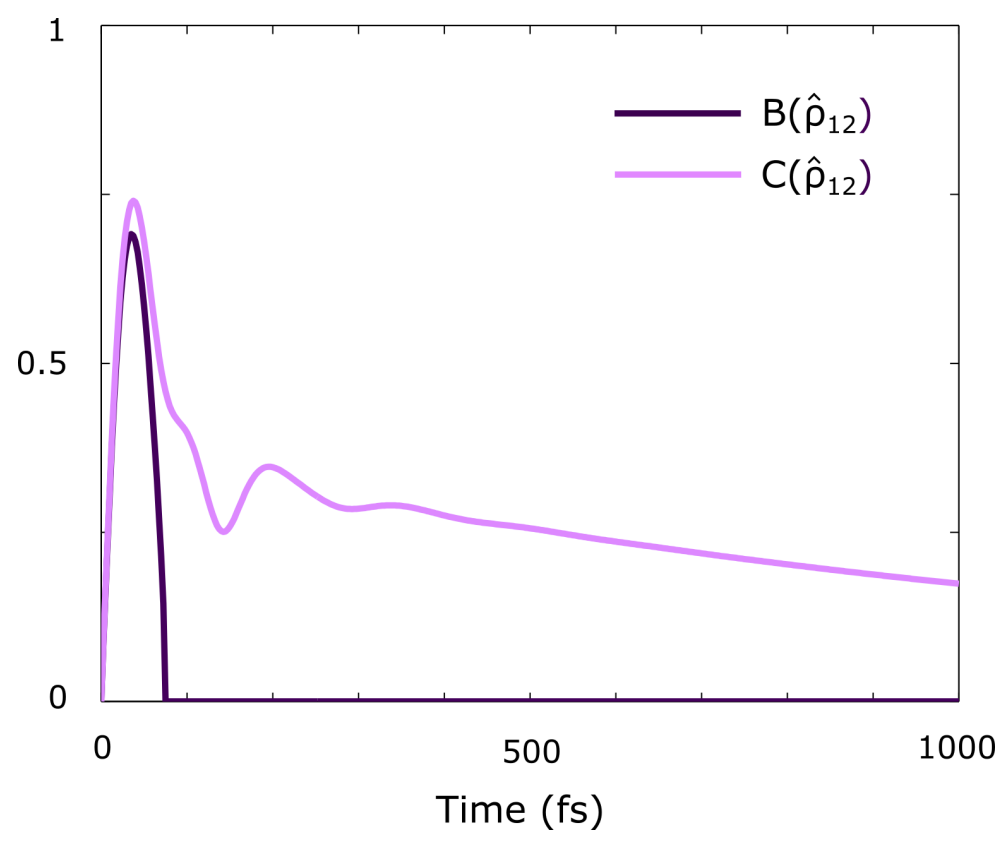

Figure 5: Nonlocality $B\left(\hat{\rho}_{12}\right)$ and entanglement $C\left(\hat{\rho}_{12}\right)$ for the chromophore pair 1 and 2 as a function of time when chromophore 1 is initially excited. Note how the nonlocality drops to zero within about $80 \mathrm{fs}$.

These numerical values imply

$$
\begin{aligned}
C\left(\hat{\rho}_{12}\right) & =2\left|\varrho_{12}\right| \approx 0.428, \\
\mu_{1} & =\mu_{2}=\left[C\left(\hat{\rho}_{12}\right)\right]^{2} \approx 0.183, \\
\mu_{3} & =\left(1-2 \varrho_{11}-2 \varrho_{22}\right)^{2} \approx 0.781 ;
\end{aligned}
$$

thus, $\hat{\varrho}_{\mathrm{FRET}}^{x=1}$ shows entanglement $(C>0)$ but no nonlocality $\left(B=0\right.$ since $\mu_{p}+\mu_{q}=$ $0.183+0.781=0.964<1$ ) between the first two chromophores. Since correlations essentially are concentrated to this chromophore-pair, it follows that all chromophore pairs are locally correlated at $t=0$, given the FRET initial condition.

On the other hand, we note that the two dominating exciton states $\hat{\varrho}^{e_{3}}=\left|e_{3}\right\rangle\left\langle e_{3}\right|$ and $\hat{\varrho}^{e_{6}}=\left|e_{6}\right\rangle\left\langle e_{6}\right|$ are strongly nonlocal between chromophores 1 and 2 ; indeed, one finds

$$
\begin{aligned}
& B\left(\hat{\rho}_{12}^{e_{3}}\right)=C\left(\hat{\rho}_{12}^{e_{3}}\right) \approx 2\left|c_{31} c_{32}\right|=0.801, \\
& B\left(\hat{\rho}_{12}^{e_{6}}\right)=C\left(\hat{\rho}_{12}^{e_{6}}\right) \approx 2\left|c_{61} c_{62}\right|=0.822,
\end{aligned}
$$

where we have used that $B=C$ for pure states of any TLS-pair ${ }^{15}$. By comparing these 


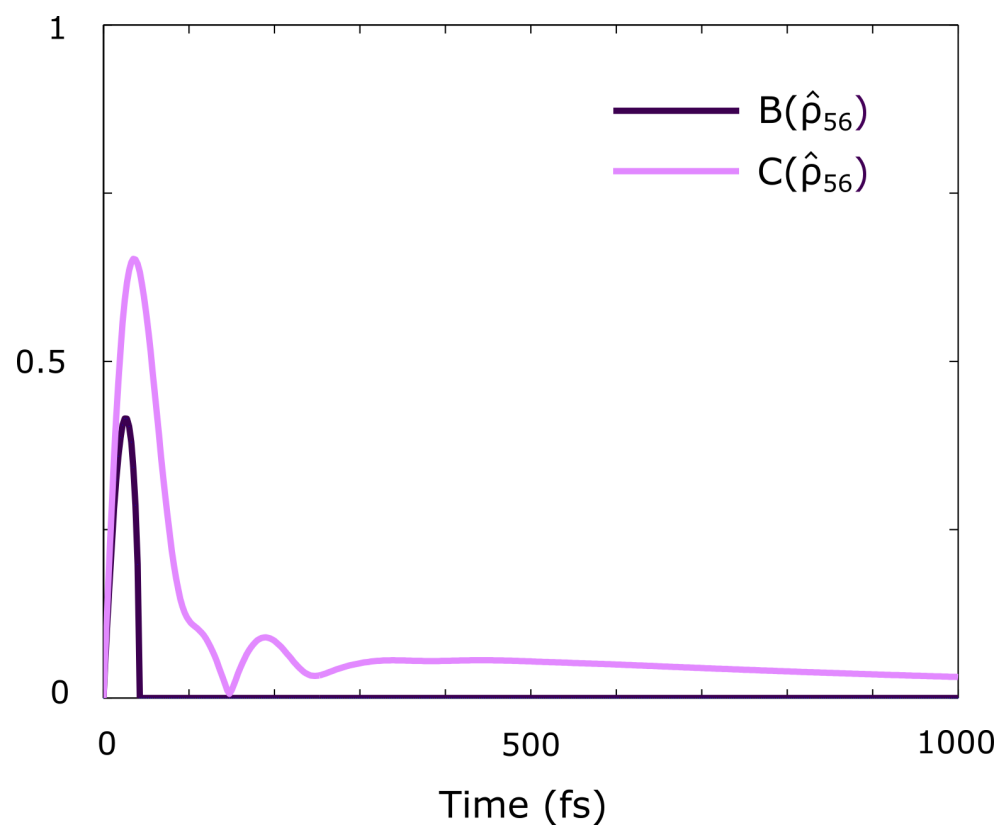

Figure 6: Nonlocality $B\left(\hat{\rho}_{56}\right)$ and entanglement $C\left(\hat{\rho}_{56}\right)$ for the chromophore pair 5 and 6 as a function of time when chromophore 6 is initially excited. Note how the nonlocality drops to zero within about $40 \mathrm{fs}$.

expressions with the expression for $\varrho_{12}$ in Eq. (35), we find

$$
\varrho_{12} \approx c_{31}^{2} B\left(\hat{\rho}_{12}^{e_{3}}\right)-c_{61}^{2} B\left(\hat{\rho}_{12}^{e}\right),
$$

which explicitly entails that the essential source for the disappearance of nonlocality when mixing the exicton state is the destructive quantum-mechanical interference (relative minus sign) between the first-site components $c_{31}$ and $c_{61}$ of $\left|e_{3}\right\rangle$ and $\left|e_{6}\right\rangle$.

A similar analysis of the $x=6$ case can be carried out with the same conclusion that all chromophore pairs are locally correlated, but entangled at $t=0$.

FRET are stationary states under the action of the electronic Hamiltonian, but they may undergo nontrivial evolution under influence of the environment. Thus, the environment is a potential source of nonlocal correlations to show up at $t>0$. However, as can be seen in Figs. 3 and 4 , it turns out that nonlocal correlations never appear given the FRET initial conditions, despite the fact that entanglement is typically nonvanishing.

Next, we consider the case of localized initial states $\varrho_{\text {localized }}^{x}=|x\rangle\langle x|, x=1,6$. These states are not eigenstates of $H_{e}$ and can therefore in principle give rise to nonlocal correlations 
during time evolution.

Let us determine for which chromophore pair(s) nonlocality can occur. In the short time limit, we may expect that $\mathbf{n}=0$ is the dominant term in the HEOM. Thus, we may assume that $\hat{\varrho}(\delta t) \approx e^{-i \delta t \hat{H}_{e}} \hat{\varrho}_{\text {localized }}^{x} e^{i \delta t \hat{H}_{e}}$ for small $\delta t$ (from now on, we put $\hbar=1$ for notational simplicity). By expanding in $\delta t$, we find to leading orders:

$$
\begin{aligned}
C\left(\hat{\rho}_{x n}\right) & \approx 2 \delta t\left|J_{x n}\right| \\
C\left(\hat{\rho}_{m n}\right) & \approx 2 \delta t^{2}\left|J_{m x} J_{x n}\right| \\
B\left(\hat{\rho}_{x n}\right) & \approx 2 \delta t \sqrt{\max \left\{\left(J_{x n}^{2}-\sum_{l \neq n} J_{x l}^{2}\right), 0\right\}}, \\
B\left(\hat{\rho}_{m n}\right) & =0,
\end{aligned}
$$

where $m, n \neq x$. Here, $J_{k l}$ are the real-valued dipole coupling parameters given by the offdiagonal terms of $H_{e}$. We may therefore draw the following conclusions about the short-time behavior of a localized initial state:

(i) entanglement between the initially excited chromophore $x$ and any other chromophore $n$ increases linearly with $\delta t$ with proportionality factor given by the corresponding dipole coupling parameter $\left|J_{x n}\right|$;

(ii) entanglement between any other pair of chromophores increases quadratically with $\delta t$;

(iii) nonlocal correlation between chromophore $x$ and chromophore $n$ increases linearly with $\delta t$ provided

$$
J_{x n}^{2}>\sum_{l \neq n} J_{x l}^{2}
$$

(iv) nonlocal correlations between any other pair of chromophores vanish.

The inequality in Eq. 40 can be satisfied for at most one pair. The explicit form of $H_{e}$ entails that only chromophores 1 and 2 can show nonlocal correlation after excitation of chromophore 1, while only chromophores 5 and 6 can show nonlocal correlation after excitation of chromophore 6. For these pairs we further notice that entanglement grows linearly with almost the same speed since $\left|J_{12}\right| \sim\left|J_{56}\right|$. As can be seen in Figs. 5 and 
6. these results are confirmed in our numerical simulations of the HEOM, where we find nonlocality for $t$ less than $\sim 80$ fs and $\sim 50$ fs for $x=1$ and $x=6$, respectively. Indeed, at these instances of time, nonlocality suddenly disappears, although entanglement persists. We call this phenomenon nonlocality sudden death, being a striking manifestation of that nonlocality and entanglement are different properties for mixed quantum states 17 .

The absence of nonlocal correlation after its sudden death for localized initial states is a consequence of open system effects. In contrast, the complete absence of nonlocality in the FRET case is a consequence of the local nature of the initial state. Thus, we may conclude that the disappearance of nonlocality has different origin for the two types of initial conditions.

As can be seen in Figs. 3.6, bipartite nonlocality and entanglement behave very differently in EET in the FMO complex, when the HEOM method is used to model the dynamics. For the FRET initial conditions, nonlocality is not found for any pairs of chromophores, while entanglement is nonvanishing. Furthermore, for the localized initial conditions, entanglement dynamics is similar to the chromophores population dynamics shown in Fig. 7, i.e., oscillating and exponentially decaying, while nonlocality does not show any oscillating features at all. Instead it drops to zero on a very short time scale, i.e., nonlocality may have a finite lifetime despite the fact that entanglement undergoes an exponential decay. This nonlocality sudden death is a phenomenon analogous to entanglement sudden death, i.e., the occurrence of finite-time entanglement in coherent systems, which has been predicted ${ }^{42}$ and observed in quantum optical systems ${ }^{43}$. Similarly, nonlocality sudden death has very recently been demonstrated in a two-photon experiment $\underline{44}$.

\section{CONCLUSIONS}

This work has contributed to the investigation of EET in the FMO complex in considering bipartite quantum nonlocality between different chromophores in addition to the bipartite quantum entanglement considered in previous studies ${ }^{5}$. While entanglement can alternatively be interpreted as measuring the coherences (in the sense of $l_{1}$ norm of coherence) in the FMO complex, we have shown that nonlocality cannot be given this interpretation, but 

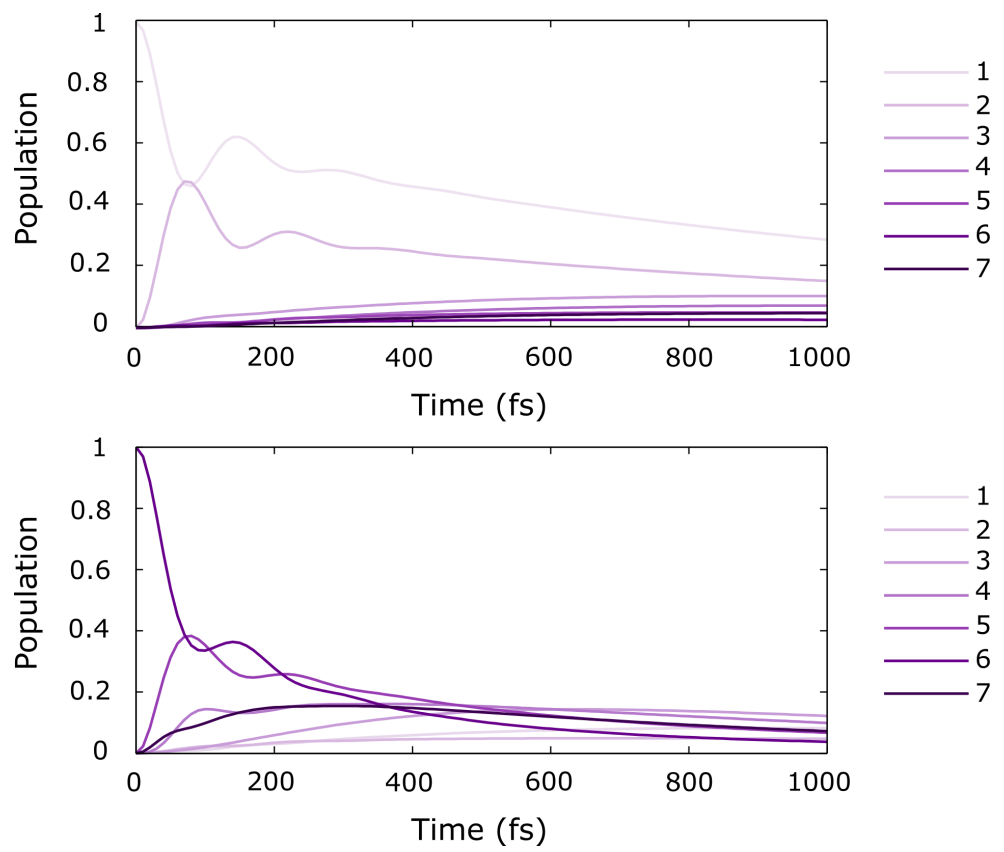

Figure 7: Population of chromophores as a function of time when chromophore 1 (upper panel) or chromophore 6 (lower panel) is initially excited. The population of the chromophores are given by the diagonal elements $\varrho_{11}, \ldots, \varrho_{77}$ of the density operator in Eq. (12).

should instead be regarded a proper quantifier of quantum correlations between chromophore pairs. The numerical simulations show that nonlocality only exists for localized initial conditions. However, it is only observed for those chromophore pairs where one of them receives the initial excitation of the system and it drops to zero on a very short time scale (less than $100 \mathrm{fs}$ ). It should be noted that the behavior of nonlocality found in our simulations is related to the fact that the FMO complex can typically exhibit only one excitation at a time; by artificially including more simultaneous excitations would potentially induce a much more complicated nonlocality pattern accompanying the EET. The restriction to the single-excitation subspace would likely prevent nonlocal multipartite correlations ${ }^{45}$ among more than two chromophores, just as the absence of genuine multipartite entanglement for this class of states, well-known from the work by Coffmann et al. $\underline{46}$.

The fact that no nonlocality is observed when more realistic initial conditions (FRET from the antenna molecules to the FMO) are used indicates that nonlocality is of no importance when considering EET in the FMO in its natural habitat. The entanglement still 
present, represents local correlations in the sense that they can be described using a theory

incorporating local realism ${ }^{177}$. Hence, the correlations between pairs of chromophores can be either quantum or classical, i.e., it cannot be ruled out that EET in the FMO complex just as well could be explained from an underlying local realistic framework. Whether this is a result of the model used in this study or actually is according to the laws of nature, remains an open question. In relation to this latter remark, we further note that to experimentally examine the behavior of nonlocality in the FMO complex would be very challenging because it would require the ability to perform local measurements in at least two different bases, which would be hard to achieve due to the short time scale and the small distances between the chromophores.

Finally, the occurence of nonlocality sudden death found in our simulations with localized initial conditions is another feature that seems to indicate that persistent quantum nonlocality is probably rare in biological systems.

\section{ACKNOWLEDGMENTS}

We thank Marie Ericsson for discussions and useful comments. M.S. acknowledges financial support from the Swedish strategic research programme eSSENCE. E.S. thanks the Swedish Research Council (VR) for financial support through Grant No. D0413201. The computations were performed on resources provided by SNIC through Uppsala Multidisciplinary Center for Advanced Computational Science (UPPMAX) under Project snic2014-3-66.

\section{References}

1. J. Adolphs, T. Renger, Biophys. J. 2006, 91, 2778.

2. G. S. Engel, T. R. Calhoun, E. L. Read, T.-K. Ahn, T. Mancal, Y.-C. Cheng, R. E. Blankenship, G. R. Fleming, Nature (London) 2007, 446, 782.

3. A. Ishizaki, G. R. Fleming, Proc. Nat. Acad. Sci. 2009, 106, 7255.

4. M. M. Wilde, J. M. McCracken, A. Mizel, Proc. Roy. Soc. A 2010, 466, 1347. 
5. M. Sarovar, A. Ishizaki, G. R. Fleming, K. B. Whaley, Nature Phys. 2010, 6, 462.

6. S. Hoyer, M. Sarovar, K. B. Whaley, New. J. Phys. 2010, 12, 065041.

7. F. Caruso, A. W. Chin, A. Datta, S. F. Huelga, M. B. Plenio, Phys. Rev. A 2010, 81, 062346 .

8. S. Hoyer, A. Ishizaki, K. B. Whaley, Phys. Rev. E 2012, 86, 041911.

9. M. B. Plenio, J. Almeida, S. F. Huelga, J. Chem. Phys. 2013, 139, 235102.

10. Y. Tanimura, R. Kubo, J. Phys. Soc. Jpn. 1989, 58, 101.

11. Y. Tanimura, Phys. Rev. A 1990, 41, 6676.

12. A. Ishizaki, G. R. Fleming, J. Chem. Phys. 2009, 130, 234111.

13. J. S. Bell, Physics 1964, 1, 195.

14. E. Schrödinger, Proc. Cambridge Phil. Soc. 1935, 31, 555.

15. B. Horst, K. Bartkiewicz, A. Miranowicz, Phys. Rev. A 2013, 87, 042108.

16. R. Augusiak, M. Demianowicz, J. Tura, A. Acín, Phys. Rev. Lett. 2015, 115, 030404.

17. R. F. Werner, Phys. Rev. A 1989, 40, 4277.

18. Y. Zhang, S. Oh, F. H. Alharbi, G. Engel, S. Kais, Phys. Chem. Chem. Phys. 2015, 17, 5743.

19. C. Creatore, M. A. Parker, S. Emmott, A. W. Chin, Phys. Rev. Lett. 2013, 111, 253601.

20. G. Panitchayangkoon, D. Hayes, K. A. Fransted, J. R. Caram, E. Harel, J. Wen, R. E. Blankenship, G. S. Engel, Proc. Nat. Acad. Sci. 2010, 10\%, 12766.

21. J. Wen, H. Zhang, M. L. Gross, R. E. Blankenship, Proc. Nat. Acad. Sci. 2009, 106, 6134 .

22. R. Pearlstein, R. P. Hemenger, Proc. Natl. Acad. Sci. 1978, 75, 4920. 
23. J. Huh, S. K. Saikin, J. C. Brookes, S. Valleau, T. Fujita, A. Aspuru-Guzik, J. Am. Chem. Soc. 2014, 136, 2048.

24. J. Martiskainen, J. Linnanto, V. Aumanen, P. Myllyperkiö, J. Korppi-Tommola, Photochem. Photobiol. 2012, 88, 675.

25. M. Ø. Pedersen, J. Linnanto, N.-U. Frigaard, N. C. Nielsen, M. Miller, Photosynth. Res. 2010, 104, 233 .

26. R. de J. León-Montiel, I. Kassal, J. P. Torres, J. Phys. Chem. B 2014, 118, 10588.

27. A. Ishizaki, T. R. Calhoun, G. S. Schlau-Cohen, G. R. Fleming, Phys. Chem. Chem. Phys. 2010, 12, 7319.

28. A. Shabani, M. Mohseni, H. Rabitz, S. Lloyd, Phys. Rev. E 2012, 86, 011915.

29. A. Einstein, B. Podolsky, N. Rosen, Phys. Rev. 1935, 47, 777.

30. J. F. Clauser, M. A. Horne, A. Shimony, R. A. Holt, Phys. Rev. Lett. 1969, 23, 880.

31. R. Horodecki, P. Horodecki, M. Horodecki, Phys. Lett. A 1995, 200, 340.

32. B. S. Cirel'son, Lett. Math. Phys. 1980, 4, 83.

33. W. K. Wootters, Phys. Rev. Lett. 1998, 80, 2245.

34. C. H. Bennett, D. P. DiVincenzo, J. A. Smolin, W. K. Wootters, Phys. Rev. A 1996, 54,3824 .

35. J. Åberg, Arxiv:quant-ph/0612146 2006.

36. T. Baumgratz, M. Cramer, M. B. Plenio, Phys. Rev. Lett. 2014, 113, 140401.

37. R. de J. Léon-Montiel, A. Vallés, H. M. Moya-Cessa, J. P. Torres, Laser Phys. Lett. 2015, 12, 085204.

38. E. L. Read, G. S. Schlau-Cohen, G. S. Engel, J. Wen, R. E. Blankenship, G. R. Fleming, Biophys. J. 2008, 95, 847. 
39. M. H. Cho, H. M. Vaswani, T. Brixner, J. Stenger, G. R. Fleming, J. Phys. Chem. B 2005, 109, 10542.

40. J. R. Dormand, P. J. Prince, J. Comp. Appl. Math. 1980, 6, 19.

41. M. A. Nielsen, I. L. Chuang, Quantum Computation and Quantum Information, Cambridge University Press, Cambridge, 2000, pp. 403-409.

42. T. Yu, J. H. Eberly, Phys. Rev. Lett. 2004 93, 140404.

43. M. P. Almeida, F. de Melo, M. Hor-Meyll, A. Salles, S. P. Walborn, P. H. Souto Ribeiro, L. Davidovich, Science 2007, 316, 579 .

44. B.-H. Liu, X.-M. Hu, J.-S. Chen, C. Zhang, Y.-F. Huang, C.-F. Li, G.-C. Guo, G. Karpat, F. F. Fanchini, J. Piilo, S. Maniscalco, arXiv:1603.09119 2016.

45. M. Żukowski and Č. Brukner, Phys. Rev. Lett. 2002, 88, 210401.

46. V. Coffmann, J. Kundu, W. K. Wootters, Phys. Rev. A 2000, 61, 052306. 